\title{
Construction of Four-Dimensional Fermionic String Models
}

\author{
Hikaru Kawai, David C. Lewellen, and S.-H. Henry Tye \\ Newman Laboratory of Nuclear Studies, Cornell University, Ithaca, New York 14853 \\ (Received 23 June 1986)
}

\begin{abstract}
We present a simple set of rules for constructing ultraviolet-finite closed-fermionic-string models. In particular, the method easily gives four-dimensional models which possess $N=1$ supersymmetry, chiral fermions, and phenomenologically interesting gauge groups.

PACS numbers: $11.17 .+\mathrm{y}, 12.10 . \mathrm{Gq}$
\end{abstract}

Superstring models are promising candidates for unified theories, in particular providing an ultravioletfinite quantum gravity. ${ }^{1}$ To date, however, the phenomenologically interesting models ${ }^{2}$ have been constructed in ten-dimensional space-time, leaving the difficult dynamical problem of compactification of the extra dimensions to be settled before any meaningful comparisons with low-energy physics can be made. It is clearly desirable to construct four-dimensional string models directly. For most low-energy physics, such a four-dimensional string model reduces to an effective-gauge-field theory with (super)gravity. Since each model has only two parameters, e.g., Newton's constant and a gauge coupling (which may be dynamically fixed), and properties of gauge theories are relatively well understood, confrontation with experiments is direct and in principle straightforward. In this Letter, we present rules for constructing consistent closed-fermionic-string theories in four dimensions. These rules are simple enough that they may be readily used even by readers not well versed in string theory. A priori, the size of the gauge groups obtained, typically rank 22 , is big enough to permit left-right symmetry, horizontal symmetries, hypercolor compositeness, and/or hidden sectors.

The only compactifications which are presently well understood at the string level are flat toroidal ones, for example that used in constructing the heterotic string. ${ }^{2}$ The possibilities for such constructions are limited by the requirements of unitarity and space-time Lorentz invariance. In particular, invariance under discrete reparametrizations of the world sheet (modular transformations) is a key ingredient in the ultraviolet finiteness of string theories and provides a stringent constraint on string-model building. In addition to these requirements one must demand that the theory give a sensible projection onto the subspace of physical states with proper space-time spin statistics.

In a previous work ${ }^{3}$ we developed a formalism based on a generalization of the projection of Gliozzi, Scherk, and $\mathrm{Olive}^{4}$ for systematically constructing ten-dimensional closed-fermionic-string models which satisfy the above requirements. Strictly speaking we imposed modular invariance only at the one-loop level; the work of Seiberg and Witten ${ }^{5}$ and others sug- gest, however, that this, together with the proper projection of physical states with correct statistics which we demand, is sufficient to ensure multiloop modular invariance. Hence we believe that the method described here generates the complete set of ultravioletfinite closed-fermionic-string models. ${ }^{6}$

Here we present the results of a straightforward generalization of the methods described in Ref. 3 to include twisted boundary conditions for complex fermionic fields on the world sheet and to allow any even space-time dimension up to ten, in particular four. As in Ref. 3 we use fermionic fields on the string world sheet to represent the internal-symmetry degrees of freedom of the string and the Neveu-Schwarz-Ramond formalism $^{7}$ for the space-time fermions. Our main concern is to ensure the modular invariance of the one-loop path integral (which is just the partition function) obtained from integrating out the fermionic degrees of freedom (viewing the string as a two-dimensional field theory on a world sheet with toroidal topology). The contribution to the partition function depends on the boundary conditions chosen for the fermionic fields around the two noncontractible loops of the world-sheet torus. In general, to achieve a modular invariant result we must sum over contributions with several different choices of boundary conditions since modular transformations can map one set of boundary conditions into another.

The rules we give here allow for twisted boundary conditions. In a complex diagonal basis for the fermionic fields the boundary conditions around the $\sigma$ loop of the torus, for example, can be specified by a single vector $\mathbf{W}$ with rational numbers as components $\left(0 \leqslant W^{l}<1\right)$,

$$
\Psi^{\prime}(t, \sigma+2 \pi)=\exp \left(2 \pi i W^{l}\right) \Psi^{\prime}(t, \sigma),
$$

where $/$ labels the complex fermionic fields for internal and space-time degrees of freedom, both left- and right-moving modes. Similarly some other vector $\mathbf{W}^{\prime}$ labels the set of boundary conditions in the $t$ direction. The fields $\Psi^{l}$ are generalizations of Neveu-SchwarzRamond fermions and have been analyzed by Vafa. ${ }^{8}$ For simple boundary conditions (periodic or antiperiodic), complex fermion fields can be decomposed into real fields. The "single-fermion" cases are of in- 
terest because they permit the reduction of the total rank of the gauge group ${ }^{3}$ as well as the construction of models in both even and odd space-time dimensions. Details of the general analysis will be discussed in Kawai, Lewellen, and Tye, ${ }^{9}$ which also contains a discussion of string interactions and other related topics.

For a fixed set of $\sigma$ boundary conditions a sum over contributions to the partition function with different $t$ boundary conditions naturally forms a projection onto a subspace of string states. ${ }^{3-5}$ In addition to requiring that the sum over contributions from different bound- ary conditions give a modular invariant partition function we demand that the projection produced be sensible, i.e., have eigenvalues 0 and 1 (so that a string state is either included in the partition function or is not), and include physical states with the correct space-time statistics, i.e., space-time bosons should contribute to the one-loop amplitude with a positive overall sign, while space-time fermions should contribute with an additional minus sign. The general solution for the partition function (from the fermionic degrees of freedom) satisfying these conditions is ${ }^{9}$

$$
\begin{aligned}
Z_{\text {fermion }}=\left(\prod m_{i}\right)^{-1} \sum_{\left\{\alpha_{i}, \boldsymbol{\beta}_{i}\right\}} \exp \left\{2 \pi i \left[\sum_{i}\left(\beta_{i}+\delta_{i 0}\right)\left(\sum_{j} k_{i j} \alpha_{j}-\mathbf{W}_{i} \cdot \overline{\alpha \mathbf{W}}+k_{0 i}+S_{i}\right)\right.\right. \\
\left.\left.+\alpha S-\frac{1}{2}\right]\right\} \operatorname{Tr}\left(\exp \left\{2 \pi i\left[\tau \hat{H}_{\alpha W}^{\text {left }}-\bar{\tau} \hat{H}_{\alpha W}^{\text {right }}-\Sigma_{j}\left(\beta_{j}+\delta_{j 0}\right) \mathbf{W}_{j} \cdot \hat{\mathbf{N}}_{\alpha W}\right]\right\}\right),
\end{aligned}
$$

with the constraints ( $i, j$ not summed),

$$
\begin{aligned}
& k_{i j}+k_{j i}=\mathbf{W}_{i} \cdot \mathbf{W}_{j}(\bmod 1), \\
& m_{j} k_{i j}=0(\bmod 1), \\
& k_{i i}+k_{i 0}+S_{i}-\frac{1}{3} \mathbf{W}_{i} \cdot \mathbf{W}_{i}=0(\bmod 1) .
\end{aligned}
$$

Here the structure constants $k_{i j}$ are rational numbers. The vectors specifying the boundary conditions represented in the sum form a vector space spanned by basis vectors $\mathbf{W}_{i}$. The boundary conditions in the $\sigma$ and $t$ directions are given by $\overline{\alpha \mathbf{W}}$ and $\overline{\beta \bar{W}}$, respectively, where $\overline{\alpha \mathbf{W}}=\Sigma_{i} \alpha_{i} \mathbf{W}_{i}$. The coefficients $\alpha_{i}$ and $\beta_{i}$ take integer values from 0 to $m_{i}-1$, where $m_{i}$ is the smallest integer such that all of the components of $m_{i} \mathbf{W}_{i}$ ( $i$ not summed) are integers. The set of $\mathbf{W}_{i}$ must be independent, i.e., $\overline{\alpha \mathbf{W}}=0$ implies that all $\alpha_{i}=0$. The sum in (2) runs over all values of all the $\alpha$ and $\beta$ coefficients. The overbar signifies that we include only the fractional part of each component of the vector $\alpha \mathbf{W}$, i.e., while $\alpha W^{l}$ can be greater than 1 we always have $0 \leqslant \alpha W^{l}<1$. The dot product involving $\mathbf{W}_{i}$ in (2)-(5) is defined to be $\mathbf{W}_{i} \cdot \mathbf{W}_{j}=\Sigma_{l} \epsilon(l) W_{i}^{l} W_{j}^{l}$, where $\epsilon(l)$ is 1 for $l$ labeling left-moving fermionic fields and -1 for $l$ labeling right-moving fermionic fields. This structure arises because the contribution of a right-moving fermionic field to the partition function is obtained from the contribution of a left-moving fermionic field by complex conjugation. The vector $\mathbf{W}_{0}$ has all components equal to $\frac{1}{2}$ and must be present in the vector space to ensure a proper projection. ${ }^{3} \alpha S$ $=\Sigma \alpha_{i} S_{i}=0$ or $\frac{1}{2}$ (depending on the space-time components of $\overline{\alpha \mathbf{W}}$ ) represents a space-time fermion (Ramond sector) or boson (Neveu-Schwarz sector), respectively. For heterotic strings, $S_{i} \equiv W_{i}^{0}$.

The $\tau$ appearing in (2) is the complex modular parameter of the world-sheet torus. $\hat{H}_{\alpha W}^{\text {left }}\left(\hat{H}_{\alpha W}^{\text {right }}\right)$ is the Hamiltonian operator for the left-moving (rightmoving) fermions with $\sigma$ boundary conditions specified by $\overline{\alpha \mathbf{W}}$. It consists of a sum over oscillator modes with quanta $q-\overline{\alpha W^{l}}, q$ a positive integer, for antifer- mions and $q+\overline{\alpha W^{l}}-1$ for fermions. For each complex fermion field, $\hat{H}_{\alpha W}$ also includes a vacuum energy,

$$
E^{l}=\frac{1}{2}\left[\left(\overline{\alpha W^{l}}\right)^{2}-\overline{\alpha W^{l}}+\frac{1}{6}\right] .
$$

The components of $\hat{\mathbf{N}}_{\alpha W}$ are the fermion number operators (number of fermions minus number of antifermions) for each of the complex fermion fields, and as before, the dot product with $\mathbf{W}_{i}$ includes an additional factor of -1 for the right-moving modes.

The phase in front of the trace in (2) is constructed, together with the constraints $(3)-(5)$, so that $Z$ is invariant under modular transformations of $\tau$. The sum over the $\beta$ coefficients forms a projection operator, as desired, with only those states contributing to $Z$ which satisfy

$$
\begin{aligned}
\mathbf{W}_{i} & \cdot \mathbf{N}_{\alpha W} \\
& =S_{i}-\mathbf{W}_{i} \cdot \overline{\alpha \mathbf{W}}+\sum_{j} k_{i j} \alpha_{j}+k_{0 i}(\bmod 1) .
\end{aligned}
$$

The physical states allowed by this constraint contribute to the partition function $Z$ with an overall phase $\exp \left\{2 \pi i\left[\alpha S-\frac{1}{2}\right]\right\}$, giving the correct physical spacetime statistics, regardless of the particulars of the internal symmetry.

Equations (3) through (7) provide a set of rules for constructing the spectra of consistent closed-fermionic-string models, without further reference to Eq. (2) and the details of string theories which it represents. First one must choose a set of basis vectors $\mathbf{W}_{i}$ of the appropriate form for the space-time dimension and type of string model (type II or heterotic) desired (examples will follow). The components of these vectors and the constants $k_{i j}$ must be chosen consistent with (3)-(5). Each specific choice defines an acceptable model. Then the spectrum-generating formula (7) provides a set of equations for the allowed fermion numbers in a given physical sector characterized by $\overline{\alpha W}$. The physical particle content of the model is gen- 
erated by consideration of all allowed sets of $\alpha_{i}$. The left- or right-moving quanta in the first-quantized theory are added to the appropriate vacuum energy to build up the (mass) ${ }^{2}$ of a physical particle excitation subject to the constraint that the (mass) ${ }^{2}$ calculated in the left- and right-moving sectors of the theory are identical. Explicitly, for each sector $\overline{\alpha \mathbf{W}}, m^{2}=m^{2}($ left $, n, \bar{n})=m^{2}($ right $, n, \bar{n})$, where

$$
m^{2}(\text { left }, n, \bar{n})=\sum_{l(\text { left }), q}\left\{\left(q-\overline{\alpha W^{l}}\right) \bar{n}_{q}^{l}+\left(q+\overline{\alpha W^{l}}-1\right) n_{q}^{l}\right\}+\sum_{l(\text { left })} E^{l}-[(D-2) / 24]+\sum_{q} q M_{q},
$$

and similarly for $m^{2}$ (right, $\left.n, \bar{n}\right)$. Here we have

$$
N_{\alpha W}^{\prime}=\sum_{q} n_{q}^{l}-\bar{n}_{q}^{l} \text {. }
$$

The sum in (8) runs over $l$ labeling left-moving fermion fields, $n_{q}^{l}$ and $\bar{n}_{q}^{l}$ are fermion and antifermion occupation numbers for the individual excitation modes in the $\overline{\alpha \mathbf{W}}$ sector, and $D$ is the space-time dimension [the $(D-2)$ term is the contribution of the string coordinates in light-cone gauge to the vacuum energy]. $M_{q}$ is the excitation number for the integral quanta $q$ of the string coordinates. The units in (8) are $8 \pi$ times the string tension; for low-energy particle content only the massless sector is relevant, others being of the order of the Planck mass.

We will sketch the use of this formalism by way of a few simple examples. Consider first the ten-dimensional heterotic-type theory. There are four rightmoving complex fermionic fields and sixteen leftmoving ones [these numbers chosen to cancel the (super)conformal anomaly]. The four right movers all have the same boundary conditions. This is required for local world-sheet supersymmetry of the covariant theory, needed in turn to decouple the timelike fermionic modes. ${ }^{3}$ Since these represent space-time degrees of freedom we have $S_{i}=W_{i}=0$ or $\frac{1}{2}$ for $l=0$ to 3 (type-II theories have this structure for the left movers as well). In an obvious notation (the bar separating right and left movers) we have $\mathbf{W}_{0}$ $=\left(\left(\frac{1}{2}\right)^{4} \mid\left(\frac{1}{2}\right)^{16}\right)$. If we choose this as the only basis vector, then the 16 complex left-moving fields are equivalent to 32 real ones with a natural $\mathrm{SO}(32)$ symmetry group. The model has two sectors, $\mathbf{W}_{0}$ and the zero vector $\mathbf{0}$. For the $\mathbf{W}_{\mathbf{0}}$ sector, the ground-state energy level is given by $(8)$ to be $\left(-\frac{1}{2},-1\right)$ for (right, left) movers and (7) gives $\frac{1}{2} \sum_{1} N_{W_{0}}^{l}=\frac{1}{2}(\bmod 1)$ for either allowed value of $k_{00}=0, \frac{1}{2}$. For $W^{l}=\frac{1}{2}$, the lowest excitation raises the level by $\frac{1}{2}$ unit. Choosing any one of the left-moving sector $n_{1}^{\prime}$ or $\bar{n}_{1}^{\prime}=1$ satisfies (8). The 32 choices yield tachyonic states $\left(m^{2}=-\frac{1}{2}\right)$ transforming as an $\mathrm{SO}(32)$ vector. To get massless bosons, we excite a right-moving mode with quantum $\frac{1}{2}$, yielding a space-time vector index, and in the leftmoving sector require $\Sigma_{l \text { (left) }} n_{1}^{l}+\bar{n}_{1}^{l}=2$. This gives the 496 gauge bosons in the adjoint representation. Taking $M_{1}=1$ gives the gravity multiplet. The zero vector 0 sector (i.e., $\alpha=0$ ) has ground-state level $(0,1)$ and hence produces only massive fermions. This is the SO(32) tachyonic model. ${ }^{5,10}$
Including $\mathbf{W}_{1}=\left((0)^{4} \mid\left(\frac{1}{2}\right)^{16}\right)$ as a basis vector adds one more constraint from (7) which serves to eliminate the tachyons from the spectrum and adds another massless physical sector which produces 496 fermions in the adjoint representation of $\mathrm{SO}(32)$ to give the SO(32) heterotic string model. ${ }^{2}$ The ground state of the periodic components $\left(W_{1}^{l}=0, \quad l=0\right.$ to 3$)$ transforms as an $\mathrm{SO}(8)$ spinor to give the space-time spinors [fermion number 0 or 1 signifies which of the two $S O(8)$ spinor representations and hence left or right space-time chirality]. Adding $\mathbf{W}_{2}=\left((0)^{4} \mid\right.$ $\left.\left(\frac{1}{2}\right)^{8}(0)^{8}\right)$ to the basis breaks the $\mathrm{SO}(32)$ group to $\mathrm{SO}(16) \otimes \mathrm{SO}(16)$. If $k_{12}$ is taken to be $\frac{1}{2}$ then there are no massless particles from the physical sectors given by $\mathbf{W}_{0}+\mathbf{W}_{2}$ or $\mathbf{W}_{0}+\mathbf{W}_{1}+\mathbf{W}_{2}$ [with ground level $\left.\left(-\frac{1}{2}, 0\right)\right]$ and the gauge group is in fact $\operatorname{SO}(16)$ $\otimes \operatorname{SO}(16){ }^{10,11}$ If $k_{12}=0$ then these two sectors give (with fermion number 0 or 1) gauge bosons in the $(128,1)$ and $(1,128)$ representation of $\mathrm{SO}(16)$ $\otimes$ SO $(16)$ which taken together with the $(1,120)$ and $(120,1)$ from the $\mathbf{W}_{0}$ sector give the gauge group $E_{8} \otimes E_{8}{ }^{2}$ In general, different choices of $\mathbf{W}_{i}$ and $k_{i j}$ can give the same physical model; for example, the basis $\mathbf{W}_{0}$ and $\mathbf{W}_{2}$ gives the $E_{8} \otimes S O(16)$ tachyonic model $^{3,10}$ for any allowed choice of $k_{i}$, as does the basis $\mathbf{W}_{0}, \mathbf{W}_{2}$, and $\mathbf{W}_{3}=\left((0)^{4} \mid\left(\frac{1}{2}\right)^{6}(0)^{2}\left(\frac{1}{2}\right)^{2}(0)^{6}\right)$.

In constructing four-dimensional models six bosonic and six real fermionic space-time degrees of freedom of the ten-dimensional string become internal. To apply our formalism the six internal bosonic degrees of freedom are fermionized. For the left-moving sector, they simply increase the number of complex fermions from 16 to 22 . For the right-moving sector, each of the six internal boson fields plus its fermionic superpartner becomes an SO(3) triplet of real fermions. Together they have an $[\mathrm{SO}(3)]^{6} \otimes S_{6}$ (permutation) symmetry. It is straightforward to check that this fermionization gives the super-Virasoro algebra with the correct central charge. Writing the six triplets as three triplets of complex fermions, the general form of the $\mathrm{W}_{i}$ is ${ }^{9}$

$$
\begin{aligned}
& \mathbf{W}_{i}=\left(S_{i}\left(a_{1} b_{1} c_{1}\right)\left(a_{2} b_{2} c_{2}\right)\left(a_{3} b_{3} c_{3}\right) \mid d_{1} \cdots d_{22}\right), \\
& a_{l}+b_{l}+c_{l}=S_{i}(\bmod 1) .
\end{aligned}
$$

Here $S_{i}=0$ or $\frac{1}{2}$ is the only space-time component and the constraint on the triplet structure for the right movers is again a consequence of the local world-sheet 
supersymmetry of the covariant two-dimensional theory.

As before we always have the vector $\mathbf{W}_{0}$ $=\left(\left(\frac{1}{2}\right)^{10} \mid\left(\frac{1}{2}\right)^{22}\right)$. The vacuum energy level of the (right, left) movers is $\left(-\frac{1}{2},-1\right)$. Taken by itself this gives a tachyonic model with gauge group $[\mathrm{SO}(3)]^{6}$ $\otimes$ SO (44) and no massless fermions. The SO(44) symmetry arises naturally from the left movers; the [SO(3) $]^{6}$ gauge bosons are obtained by taking $M_{1}=1$ for the left movers (to get a space-time vector) and exciting the right-moving internal degrees of freedom. Adding $\mathbf{W}_{1}=\left(0\left(0 \frac{1}{2} \frac{1}{2}\right)^{3} \mid\left(\frac{1}{2}\right)^{22}\right)$ removes the tachyons and adds a sector of massless fermions, in fact giving an $N=4$ supersymmetric $S O(44)$ model. ${ }^{12}$ All that remains of the $[\mathrm{SO}(3)]^{6}$ symmetry are the six vector bosons in the $N=4$ supergravity multiplet. In general, nonsupersymmetric models are obtained if $\mathbf{W}_{1}$ is excluded. We can reduce the supersymmetry and/or break the gauge group by adding more basis vectors. Including, for example, $\mathbf{W}_{2}=\left(0\left(0 \frac{1}{2} \frac{1}{2}\right)\left(\frac{1}{2} 0 \frac{1}{2}\right)^{2} \mid\right.$. $\left.\left(\frac{1}{2}\right)^{14}(0)^{8}\right)$, we can choose $k_{i j}$ so that we obtain an $N=2$ supersymmetric $\mathrm{SO}(28) \otimes \mathrm{SO}(16)$ model. Addition of

$$
\mathbf{W}_{3}=\left(0\left(\frac{1}{2} 0 \frac{1}{2}\right)\left(0 \frac{1}{2} \frac{1}{2}\right)\left(\frac{1}{2} \frac{1}{2} 0\right) \mid\left(\frac{1}{2}\right)^{7}(0)^{7}\left(\frac{1}{2}\right)^{3}(0)^{5}\right)
$$

to this set produces, for some choices of $k_{i j}$, an $N=1$ supersymmetric model with the gauge group $\mathrm{SO}(14)$ $\otimes S O(14) \otimes S O(6) \otimes S O(10)$. In addition to gauginos in adjoint representations and fermions in vector representations of the gauge group [from the $\mathbf{W}_{1}$ sector, e.g., $(14,14,1,1),(14,1,6,1),(1,14,1,10)$, etc.], one can check in this case that the physical sector given by $\mathbf{W}_{2}$ [with ground level $(0,0)$ ] yields two chiral fields in the $(4,16)$ and $(\overline{4}, 16)$ representations of $\mathrm{SO}(6) \otimes S O(10)$. If $\mathrm{SO}(6)$ is taken as a horizontal symmetry group, this model has sixteen chiral families in $\mathrm{SO}(10)$. We note that a scalar supermultiplet always accompanies the $N=1$ supergravity multiplet.

The group structure in four dimensions obtainable from our rules is very rich. For example, choosing $\mathbf{W}_{0}$ and $\mathbf{W}_{1}$ from above and

$$
\begin{aligned}
& \mathbf{W}_{2}=\left(0\left(0 \frac{1}{2} \frac{1}{2}\right)\left(\frac{1}{2} 0 \frac{1}{2}\right)^{2} \mid(0)^{16}\left(\frac{1}{2}\right)^{6}\right), \\
& \mathbf{W}_{3}=\left(0\left(\frac{1}{2} \frac{1}{2} 0\right)^{2}\left(0 \frac{1}{2} \frac{1}{2}\right) \mid\left(\frac{1}{2}\right)^{2}(0)^{3}\left(\frac{1}{4}\right)^{8}\left(\frac{1}{3}\right)^{9}\right),
\end{aligned}
$$

we can obtain an $N=1$ supersymmetric model with the gauge group $E_{7} \otimes \mathrm{SU}(2) \otimes \mathrm{U}(8) \otimes \mathrm{SO}(4)$ $\otimes \mathrm{SO}(8)$. Also, $N=1$ models can be obtained from type-II strings. The gauge group obtained from our method can be precisely identified. If we keep track of the $U(1)$ charges from each complex fermionic field $\left(\alpha W^{l}-\frac{1}{2}+N_{\alpha W}^{l}\right.$ for the $l$ th charge in the $\overline{\alpha \mathbf{W}}$ sector), each massless vector boson corresponds to a root vector in Cartan's construction of a Lie group. It can be shown that the gauge bosons arising in our construction always transform as the adjoint representation of a simply laced Lie group. ${ }^{9}$

Finally we note that it will be interesting to clarify the close connection of this work with the idea of strings on orbifolds. ${ }^{13}$

This work is supported by the National Science Foundation.

${ }^{1}$ M. B. Green and J. H. Schwarz, Nucl. Phys. B255, 93 (1985); for a review see, e.g., J. H. Schwarz, Phys. Rep. 89, 223 (1982); M. B. Green, Surv. High Energy Phys. 3, 127 (1983)

2D. J. Gross, J. A. Harvey, E. Martinec, and R. Rohm, Phys. Rev. Lett. 54, 502 (1985), and Nucl. Phys. B256, 253 (1985), and B267, 75 (1986).

${ }^{3}$ H. Kawai, D. C. Lewellen, and S.-H. H. Tye, Phys. Rev. $\mathrm{D}$ (to be published).

${ }^{4}$ F. Gliozzi, J. Scherk, and D. Olive, Nucl. Phys. B122, 253 (1977).

${ }^{5} \mathrm{~N}$. Seiberg and W. Witten, to be published.

${ }^{6}$ That is, if we also include Thirring interactions for the world-sheet fermions. See H. Kawai, D. C. Lewellen, and S.-H. H. Tye, to be published.

${ }^{7}$ P. Ramond, Phys. Rev. D 3, 2415 (1971); A. Neveu and J. H. Schwarz, Nucl. Phys. B31, 86 (1971).

${ }^{8} \mathrm{C}$. Vafa, Harvard University Report No. HUTP-86/A011 (to be published).

${ }^{9}$ Kawai, Lewellen, and Tye, Ref. 6.

${ }^{10}$ L. J. Dixon and J. A. Harvey, to be published.

${ }^{11}$ L. Alvarez-Gaume, P. Ginsparg, G. Moore, and C. Vafa, Harvard University Report No. HUTP-86/A013 (to be published)

${ }^{12}$ K. S. Narain, Phys. Lett. 169B, 41 (1986).

${ }^{13}$ L. Dixon, J. Harvey, C. Vafa, and E. Witten, Nucl. Phys. B261, 678 (1985), and to be published. 